\title{
STATUS AND PROSPECTS OF $e^{+} e^{-}$LINEAR COLLIDER PROJECTS*
}

\author{
SABine RiEmann \\ Deutsches Elektronen-Synchrotron, DESY \\ Platanenallee 6, 15738 Zeuthen, Germany
}

(Received October 21, 2015)

This article gives a short overview of the ILC and CLIC $e^{+} e^{-}$linear collider projects and reviews key motivations of future $e^{+} e^{-}$collider projects.

DOI:10.5506/APhysPolB.46.2213

PACS numbers: 29.20.Ej, 13.66.Jn

\section{Introduction}

The discovery of the Higgs boson in 2012 [1] completed the particle spectrum of the Standard Model (SM). The Higgs boson mass was found in the range predicted by electroweak measurements, and so far, all properties of the Higgs boson are consistent with those of an SM Higgs boson. However, the final conclusion whether it is really the SM Higgs boson requires the precise measurement of its parameters, in particular the Higgs coupling to bosons and fermions. But the focus is also on phenomena beyond the SM, for example the existence of dark matter, which must be understood and embedded in a theory. During the next years, the LHC will continue its physics programme at highest energies and high luminosities. Nevertheless, analyses independent of model assumptions are hard at the LHC. Despite the excellent performance, the measurements at the LHC are not sensitive to all phenomena. High-energy $e^{+} e^{-}$colliders provide complementary information which allows to establish a more complete picture of elementary particles and their interactions. However, in circular $e^{+} e^{-}$colliders, the energy loss by synchrotron radiation increases with $E_{\text {beam }}^{4} / R$. Operating at energies beyond $200-300 \mathrm{GeV}$ requires a very large bending radius $R$ of the ring to avoid huge losses by synchrotron light. Linear colliders overcome this problem. Here, an overview of the status of high-energy linear $e^{+} e^{-}$collider projects is given and an insight into the physics potential is provided.

* Presented at the XXXIX International Conference of Theoretical Physics "Matter to the Deepest", Ustroń, Poland, September 13-18, 2015. 


\section{Future $e^{+} e^{-}$linear collider projects}

There are two $e^{+} e^{-}$linear collider projects for energies up to the $\mathrm{TeV}$ range: The International Linear Collider (ILC) and the Compact Linear Collider (CLIC). A flexibility to operate at several energies is highly desired to cover all interesting processes. Linear $e^{+} e^{-}$colliders need high accelerating gradients to keep the collider length in reasonable limits. They require powerful electron and positron sources as well as extremely small beam cross sections at the interaction point to achieve the luminosity desired for precision measurements. Besides the problems related to the particular collider technology, the global R\&D work for $e^{+} e^{-}$accelerators and detectors and also for physics studies is organized within the Linear Collider Collaboration (LCC) which brings together the communities of ILC and CLIC.

\subsection{The International Linear Collider}

The International Linear Collider (ILC) [2] is a world-wide project with contributing labs from Europe, Asia and Americas. It is designed for centreof-mass energies from the Higgs boson production threshold $(250 \mathrm{GeV})$ up to $500 \mathrm{GeV}$ and can be upgraded to $1 \mathrm{TeV}$. Further, a running at the $Z$-boson resonance is also possible to improve the statistics achieved with LEP measurements by at least two orders of magnitude. The specific luminosity is $2 \times 10^{34} \mathrm{~cm}^{-2} \mathrm{~s}^{-1}$; this allows to collect a few $100 \mathrm{fb}^{-1}$ per year. The electron beam is polarized, $P_{e^{-}} \geq 80 \%$. The positron beam polarization is about $30 \%$ in the baseline option and can be upgraded to $60 \%$. These parameters allow to measure most of the basic processes with statistical uncertainties at the percent or even per-mille level.

The accelerator utilizes $1.3 \mathrm{GHz}$ superconducting RF cavities with an average gradient of $31.5 \mathrm{MV} / \mathrm{m}$ so that the total length of a $500 \mathrm{GeV}$ machine amounts to $31 \mathrm{~km}$. The power consumption is below $200 \mathrm{MW}$ for energies below $500 \mathrm{GeV}$ and up to $300 \mathrm{MW}$ in the case of $1 \mathrm{TeV}$. The technology for the ILC is at hand; it could be built now. The European XFEL which is under construction and will start operation in 2017 uses the same technology. The cavities for the XFEL request lower gradients of about $25 \mathrm{MV} / \mathrm{m}$. Nevertheless, it has been demonstrated that many cavities achieve higher gradients of $\approx 31 \mathrm{MV} / \mathrm{m}$, even up to $35 \mathrm{MV} / \mathrm{m}$ for individual cavities. With developments by the appropriate vendors, the gradient required for the ILC will be achieved routinely.

Another important item is the luminosity. To reach the design value for nominal luminosity at $E_{\mathrm{cm}}=500 \mathrm{GeV}$, the beam emittances must be small and the beam sizes at the collision point have to be tiny. To minimize beamstrahlung effects during the bunch crossing, so-called flat beams are necessary: $\sigma_{x}(\mathrm{rms})=470 \mathrm{~nm}, \sigma_{y}(\mathrm{rms})=7 \mathrm{~nm}$. At the test facility ATF2, 
beam sizes of $\sigma=44 \mathrm{~nm}$ were demonstrated [3]; goal is $37 \mathrm{~nm}$. Taking into account all beam parameters, the value $\sigma=44 \mathrm{~nm}$ at ATF2 corresponds to $6 \mathrm{~nm}$ at the ILC.

Currently, the realization of the ILC in Japan is under discussion. The Kitakami mountains (Iwate prefecture) with thick granite bedrock have been selected as a possible site.

\subsection{The Compact Linear Collider}

The Compact Linear Collider, CLIC [4] is a potential successor of the LHC at CERN and is planned to be operated up to energies of $3 \mathrm{TeV}$. Similar to the ILC, a specific luminosity of about $2 \times 10^{34} \mathrm{~cm}^{-2} \mathrm{~s}^{-1}$ will allow to collect a few $100 \mathrm{fb}^{-1} /$ year. The electron beam is polarized, $P \geq 80 \%$. The positron beam is unpolarized in the baseline machine and if desired, it will be polarized in the upgrade option.

The CLIC design is based on the use of normal conducting accelerating structures. It utilizes a 2-beam acceleration scheme: the radiofrequency power for the main linac is extracted from a high-intensity electron drive beam $(100 A)$ which runs parallel to the colliding beams and which has a low energy of $2.4 \mathrm{GeV}$. This drive beam is decelerated to $240 \mathrm{MeV}$, and the power is transferred by Power Extraction and Transfer Structures (PETS) to the low current $(1.2 \mathrm{~A})$ main colliding beam for acceleration. The main linac uses $100 \mathrm{MV} / \mathrm{m} 12 \mathrm{GHz}$ accelerating structures. To achieve the collision energy of $3 \mathrm{TeV}$, it will be almost $50 \mathrm{~km}$ long. The power consumption at $3 \mathrm{TeV}$ is $580 \mathrm{MW}$ at the maximum.

The CLIC technology is still under development. It has been demonstrated that an accelerating structure can reach a gradient of $105 \mathrm{MV} / \mathrm{m}$ at a pulse length of $240 \mathrm{~ns}$ and a low breakdown rate in separate high-power tests. A complete $2 \mathrm{~m}$-CLIC module has been installed at the CLIC Test Facility (CTF3) and is under test. It should be remarked that there is a rapidly increasing interest in the CLIC technology, e.g. for use in linacs for Free Electron Lasers. The CLIC project plan will accommodate the LHC results. After R\&D of accelerator and detector until 2018, the decision about the next projects at the energy frontier will be made in 2018/19.

\section{Physics at high-energy $e^{+} e^{-}$linear colliders}

So far, only an SM-like Higgs boson has been discovered coupled with the absence of other phenomena. It could be that much higher energies are necessary to find new particles or forces, or the new particles have escaped detection because of very small couplings to the known particles. New results and insights are expected with the LHC running over the next years. 
But complementary to the LHC, a high energy $e^{+} e^{-}$LC will provide measurements with excellent precision from collisions of elementary particles. The initial state - i.e. the energy, the initial particle type and its polarization - is well known. Any deviation of the measurements from the SM prediction opens a window to detect new particles or forces. The physics program for future $e^{+} e^{-}$linear colliders includes physics of the Higgs boson, $W$ - and $Z$-boson physics, the top quark and phenomena beyond the SM. Details are summarized in the physics parts of the Technical and Conceptual Design Reports for future linear colliders and reference therein $[5,6]$. Here, some of the main aspects are shortly outlined.

\subsection{Higgs physics at $e^{+} e^{-}$linear colliders}

Most likely, within achievable accuracy at the LHC, the Higgs boson will appear SM-like. So it could be the only SM Higgs boson, that could belong to an extended Higgs sector or could even be a composite state. Very precise measurements of Higgs boson production and decay processes are necessary to unravel its nature.

\subsubsection{Higgs coupling to the $Z$ boson}

The Higgs-strahlung process, $e^{+} e^{-} \rightarrow Z H$, is of particular importance for the Higgs physics at future $e^{+} e^{-}$colliders: reconstructing the $Z$ boson in lepton and quark channels, the Higgs boson becomes visible in the mass spectrum recoiling against the $Z$ boson. Without reconstructing the Higgs boson itself, its mass and coupling to the $Z$ boson can be determined in a modelindependent measurement: the peak position gives the Higgs mass which can be determined at the ILC with a relative precision better than $30 \mathrm{MeV}$. The $H Z Z$ coupling is related to the peak cross section, $\sigma_{H Z Z} \propto g_{H Z Z}^{2}$, and can be measured with a relative precision $\Delta g_{H Z Z} / g_{H Z Z}=0.6 \%$ (ILC $500 \mathrm{GeV}$ ) or $\Delta g_{H Z Z} / g_{H Z Z}=0.3 \%$ (ILC 5000, high luminosity), respectively [6, 7]. The Higgs-strahlung process is also essential to determine the invisible width and the total width of the Higgs boson.

\subsubsection{Top-quark Yukawa coupling}

In the SM, the top quark has the strongest coupling to the Higgs boson. This coupling should be measured model-independent since $g_{H t \bar{t}}$ is sensitive to physics effects beyond the SM. The threshold to measure this coupling is $E_{\mathrm{cm}}>480 \mathrm{GeV}$ but at higher energy, the precision of the topYukawa coupling measurement is substantially improved. In reference [8], it is shown that running at $E_{\mathrm{cm}}=550 \mathrm{GeV}$ instead of $E_{\mathrm{cm}}=500 \mathrm{GeV}$ reduces the relative uncertainty by a factor two for the nominal luminosity to $\Delta g_{H t \bar{t}} / g_{H t \bar{t}}=9 \%$; for the high luminosity option at $550 \mathrm{GeV}$, it is even decreased to $3 \%$. 


\subsubsection{Trilinear Higgs coupling}

The measurement of the Higgs boson self-coupling is essential for establishing the Higgs mechanism. However, also at the ILC or CLIC this measurement is very challenging. The event rates are very small, $0.2-0.3 \mathrm{fb}$ depending on the degree of beam polarization, accompanied by a huge background. Analyses [9] based on the assumed full ILC data set collected at $E_{\mathrm{cm}}=500 \mathrm{GeV}$ show that a relative precision of $27 \%$ can be achieved for the trilinear Higgs coupling. In principle, this would already be more than $3 \sigma$ evidence for the existence of the Higgs self-coupling at the SM value [6]. An upgrade to $E_{\mathrm{cm}}=1 \mathrm{TeV}$ together with $2000 \mathrm{fb}^{-1}$ would result in a relative precision of $16 \%$ or even $10 \%$ in the case of $5000 \mathrm{fb}^{-1}$ [10]. The polarization of both beams is extremely important for the measurement of the trilinear Higgs coupling to reduce the background and to enhance the cross section.

\subsection{The top quark}

The precise knowledge of the top-quark mass is indispensable for tests of the SM and beyond. For example, $\Delta m_{t}=600 \mathrm{MeV}$ corresponds to $\Delta m_{W}=$ $5 \mathrm{MeV}$ for the prediction of $m_{W}$. At the ILC, it is expected to measure $m_{W}$ to a few $\mathrm{MeV}$ what enhances significantly the sensitivity to a variety of New Physics models.

So far, the top quark has been directly studied only at hadron colliders. At $e^{+} e^{-}$colliders, the top quark is produced in an electroweak process. It is a unique capability that a $t \bar{t}$ threshold scan can be performed. At the threshold, the top-quark production is strongly enhanced due to the attractive Coulomb-like gluonic interaction but the fast decays of the top quarks prevent the formation of stable $t \bar{t}$ bound states. The remnant of the smeared-out $1 S$ peak in the $e^{+} e^{-} \rightarrow t \bar{t}$ cross section can be related to the top-quark mass and total width and the QCD couplings. The top-quark mass can be determined to about $50 \mathrm{MeV}$; the accuracy is limited by the precision of the theoretical prediction of the threshold shape. As shown in reference [11], by collecting $200 \mathrm{fb}^{-1}$ around $350 \mathrm{GeV}$, statistical errors of $17 \mathrm{MeV}$ for the top-quark mass and $26 \mathrm{MeV}$ for the width can be achieved in a 3-parameter fit.

\subsubsection{Electroweak top-quark coupling}

The linear $e^{+} e^{-}$colliders will have at least one polarized beam. The measurement of the forward-backward asymmetry for two beam polarizations allows to determine the left- and right-handed top coupling to the photon and the $Z$ boson $[12,13]$. Since the top-quark coupling is sensitive to New Physics, this measurement is essential for tests of the SM and interpretation of potential deviations from the SM predictions. With a relative uncertainty at the percent-level the $g_{\mathrm{L}}, g_{\mathrm{R}}$ measurement has a substantially higher precision at an $e^{+} e^{-}$LC than at the LHC [14]. 


\subsection{Physics beyond the Standard Model}

Precision measurements at $e^{+} e^{-}$colliders will allow to discover also phenomena far beyond the energy which is directly accessible in the particle collisions. The existence of heavier particles, new forces or compositeness should manifest itself at low energies as four-fermion contact interaction. It could show up in measurements that deviate from the SM predictions. Since the mass of the Higgs boson, of the top quark and of the $W$ boson are determined with high precision, it will be possible to disentangle and to constrain the underlying New Physics model.

Models with extra $Z$ bosons are a popular example. If such particles are discovered at the LHC, they can be also obtained at an LC. If extra $Z$ bosons are produced resonantly, they can be measured with excellent precision. If the $Z^{\prime}$ mass is too high to produce $Z^{\prime}$ bosons directly at an LC, they can be detected by event rates different from the SM prediction. Assuming lepton universality, the $Z^{\prime}$ vector and axial-vector couplings to leptons can be determined in a model-independent way and used to derive the coupling to heavy quarks. Even if the LHC cannot see the $Z^{\prime}$ since it is too heavy, high-energy $e^{+} e^{-}$colliders are sensitive to the ratio of $Z^{\prime}$ coupling and $Z^{\prime}$ mass and allow to constrain the model [15].

\subsection{Physics at the $Z$ pole}

It would be extremely interesting to perform again precision measurements at the $Z$ pole but with much higher data statistics than at LEP and SLD. The ILC plans to produce $10^{9} Z$ bosons with the GigaZ option. Alternatively, also circular $e^{+} e^{-}$collider projects are under discussion which can produce up to $10^{13} Z$ bosons (see reference [16]). Measurements at the $Z$ pole with this excellent precision combined with measurements of the $W$-boson, Higgs boson and top-quark parameters would provide an ultimate test of the SM. It could be confirmed up to higher levels of loop calculations or new models beyond the SM can be established and verified.

\section{Summary}

A high energy $e^{+} e^{-}$collider should be the next large-scale device in particle physics to explore the energy frontier. This will complement the physics results gained at the LHC, improve the understanding of the SM and phenomena beyond and so finally, establish the connection of particle physics and cosmology. Japan has expressed interest in hosting the ILC, and the $e^{+} e^{-}$linear collider community supports strongly the realization of the ILC - the most mature future $e^{+} e^{-}$project — in Japan. 


\section{REFERENCES}

[1] G. Aad et al. [ATLAS Collaboration], Phys. Lett. B 716, 1 (2012) [arXiv:1207.7214 [hep-ex]]; S. Chatrchyan et al. [CMS Collaboration], Phys. Lett. B 716, 30 (2012) [arXiv:1207.7235 [hep-ex]].

[2] C. Adolphsen et al., arXiv:1306.6328 [physics.acc-ph]; C. Adolphsen et al., arXiv:1306.6353 [physics.acc-ph].

[3] K. Kubo [ATF2 Collaboration], Proceedings of the IPAC2014, Dresden, Germany, WEZA01, http://accelconf .web.cern.ch/AccelConf/IPAC2014/papers/weza01.pdf

[4] M. Aicheler et al., CERN-2012-007, SLAC-R-985, KEK-Report-2012-1, PSI-12-01, JAI-2012-001.

[5] H. Baer et al., arXiv:1306.6352 [hep-ph]; G. Moortgat-Pick et al., Eur. Phys. J. C 75, 371 (2015) [arXiv:1504.01726 [hep-ph]]; L. Linssen et al., arXiv:1202.5940 [physics.ins-det].

[6] K. Fujii et al., arXiv:1506.05992 [hep-ex].

[7] H. Li et al. [LID Design Study Group Collaboration], arXiv:1202.1439 [hep-ex].

[8] T. Barklow et al., arXiv:1506.07830 [hep-ex].

[9] J. Tian et al. [ILD Collaboration], PoS EPS HEP2013, 316 (2013) [arXiv: 1311.6528 [hep-ph]].

[10] J. Tian, LC-REP-2013-003, http://www-flc.desy.de/lcnotes/notes/LC-REP-2013-003.pdf;

M. Kurata et al., LC-REP-2013-025, http: //www-flc.desy.de/lcnotes/notes/LC-REP-2013-025.pdf

[11] T. Horiguchi et al., arXiv:1310.0563 [hep-ex]; K. Seidel et al., Eur. Phys. J. C 73, 2530 (2013) [arXiv:1303.3758 [hep-ex]].

[12] F. Richard, arXiv:1403.2893 [hep-ph]; K. Agashe et al. [Top Quark Working Group], arXiv:1311.2028 [hep-ph].

[13] M.S. Amjad et al., arXiv:1505.06020 [hep-ex].

[14] R. Röntsch, M. Schulze, J. High Energy Phys. 1508, 044 (2015) [arXiv:1501.05939 [hep-ph]]. R. Röntsch, M. Schulze, J. High Energy Phys. 1407, 091 (2014) [Erratum ibid. 1509, 132 (2015)] [arXiv:1404.1005 [hep-ph].

[15] S. Riemann, in $2^{\text {nd }}$ ECFA/DESY Study 1998-2001, pp. 1451-1468; A. Leike, S. Riemann, Z. Phys. C 75, 341 (1997) [arXiv:hep-ph/9607306];

S. Riemann, in Proceedings of the International Conference on Linear Colliders (LCWS 2004) Ed. by H. Videau, J.-C. Brient. Paris, Ecole Polytechnique, 2005, pp. 237-241.

[16] T. Lesiak, Acta Phys. Pol. B 46, 2221 (2015), this issue. 